\title{
Eficácia da combinação fixa de timolol 0,5\% e brinzolamida $1 \%$ no tratamento do glaucoma primário de ângulo aberto e na hipertensão ocular
}

\author{
Efficacy of fixed combination of timolol 10\% \\ and Brinzolamide 1\% for primary open \\ angle glaucoma and ocular hypertension
}

Juliana Almodin', Carlos Buhler Junior², Flavia Almodin³ ${ }^{3}$ Tadeu Cvintal ${ }^{4}$

\begin{abstract}
Resumo
Objetivo: Avaliar a eficácia e conforto dos pacientes portadores de glaucoma primário de ângulo aberto (GPAA) ou hipertensão ocular (HO) em uso da combinação fixa de timolol $0,5 \%$ e brinzolamida $1 \%$. Métodos: Foram acompanhados prospectivamente 26 pacientes portadores de GPAA ou HO, num total de 50 olhos que foram instituídos a utilizarem a combinação fixa de timolol $0,5 \%$ e brinzolamida $1 \%$. As avaliações foram feitas por um único examinador por tonometria de aplanação (Goldman) em 7 e 30 dias. Os possíveis efeitos colaterais e intolerância foram descritos pelos próprios pacientes através da pergunta: "Você sentiu alguma alteração ao pingar a medicação prescrita?" Os dados foram coletados e analisados estatisticamente. Resultados: Os valores de pressão intraocular (PIO) foram significativamente menores nas avaliações de 7 e 30 dias $(p<0,001)$. A média da redução da PIO foi de $38 \%$, variando de uma média inicial de $23,8 \mathrm{mmHg}$ para 14,6 e 14,4 $\mathrm{mmHg}$ nos dias 7 e 30 , respectivamente. Dos 26 pacientes incluídos apenas 4 relataram alguma queixa ao pingar o colírio, sendo que as queixas variaram de ardência, leve queimação e embaçamento. Conclusão: A combinação fixa de timolol $0,5 \%$ e brinzolamida $1 \%$ mostrou-se eficaz no tratamento de pacientes com GPAA e HO com eficácia semelhante a da literatura e apresentou um baixo índice de efeitos desconfortáveis relatados pelos usuários da medicação.
\end{abstract}

Descritores: Timolol/administração \& dosagem; Glaucoma/quimioterapia; Combinação de medicamentos; Hipertensão ocular

\footnotetext{
'Departamento de Glaucoma do Centro de Oftalmologia Tadeu Cvintal - São Paulo (SP), Brasil;

${ }^{2}$ Residente do Terceiro Ano de Oftalmologia do Centro de Oftalmologia Tadeu Cvintal - São Paulo (SP), Brasil;

${ }^{3}$ Residente do Primeiro Ano de Oftalmologia do Centro de Oftalmologia Tadeu Cvintal - São Paulo (SP), Brasil;

${ }^{4}$ Departamento de Córnea e Diretor do Centro de Oftalmologia Tadeu Cvintal - São Paulo (SP), Brasil.
}

Departamento de Glaucoma do Centro de Oftalmologia Tadeu Cvintal - São Paulo (SP), Brasil.

Os autores declaram inexistir conflitos de interesse 


\begin{abstract}
Objective: To evaluate the efficacy and side effects of timolol $0,5 \%$ and brinzolamide $1 \%$ fixed combination in patients with primary open angle glaucoma (POAG) and ocular hypertension (OH). Methods: 50 eyes of 26 patients with POAG or $\mathrm{OH}$ were evaluated with topical therapy with fixed combination of timolol $0.5 \%$ and brinzolamide $1 \%$. The measurements with Goldmann tonometry were applied by only one ophthalmologist after 7 and 30 days on medication. The side effects were described by the patient based on the following question: "Did you feel any alteration with the prescribed drops?" The data were collected and analized statistically. Results: The intraocular pressure (IOP) was lower in 7 and 30 days $(\mathrm{p}<0.001)$. The mean reduction in IOP was $38 \%$ with a variation from 23,8 mmhg to 14.6 and 14.4 mmhg in 7 and 30 days. Four patients had side effects: burning and blurring vision were related. Conclusion: the fixed combination of timolol $0.5 \%$ and brinzolamide $1 \%$ had good results with lower IOP in the treatment of patients with POAG and $\mathrm{OH}$ just like in the literature and had few side effects.
\end{abstract}

Keywords: Timolol/administration \& dosage; Glaucoma/drug therapy; Drug combinations; Ocular hypertension

\section{INTRODUÇÃO}

$\mathbf{O}$ glaucoma é a causa mais comum de cegueira irreversível nos dias de hoje ${ }^{(1)}$. Os agentes hipotensores oculares são a única farmacoterapia disponível para seu tratamento ${ }^{(2)}$. Nas últimas décadas, tivemos grandes avanços no desenvolvimento de medicações antiglaucomatosas e as opções para a terapia combinada também aumentaram ${ }^{(3)}$. A combinação fixa representa a combinação de dois agentes hipotensores oculares num único frasco ${ }^{(4)}$. Essa combinação tem a conveniência de trazer a eficácia de dois agentes hipotensores oculares ao mesmo tempo e ainda de poder aumentar a fidelidade do paciente ao tratamento.

Atualmente no mercado temos a combinação fixa de produtos com timolol $0,5 \%$ associados à brimonidina $0,2 \%$, dorzolamida $2 \%$, travoprosta $0,004 \%$, latanoprosta $0,005 \%$ e bimatoprosta $0,03 \% .{ }^{2}$ Recentemente foi formulada a combinação fixa de timolol $0,5 \%$ e brinzolamida $1 \%$ (Azorga ${ }^{\mathrm{TM}}$,Alcon laboratórios, Inc.,Ft.Worth,TX,USA). ${ }^{(5)} \mathrm{A}$ brinzolamida é um agente inibidor da anidrase carbônica que diminui a PIO através da redução da produção do humor aquoso (HA), ela é formulada a $1 \%$ em suspensão oftalmológica (Azopt,Alcon laboratórios,Inc., Ft.Worth,TX, USA) e administrada duas a três vezes ao dia. Indicada para o tratamento do GPAA e também para a HO.

Como monoterapia ou em associação a beta bloqueadores já foi demonstrado em estudos que ela tem uma ação significante na redução da PIO ${ }^{(6)}$ Estudos também demonstram que ela tem boa tolerância pelos pacientes, além de ter um menor desconforto ocular quando comparada a outras medicações. ${ }^{(7)}$

O objetivo deste estudo é de avaliar a eficácia e conforto dos pacientes portadores de GPAA ou $\mathrm{HO}$ em uso da combinação fixa de timolol $0,5 \%$ e brinzolamida $1 \%$.

\section{Métodos}

Estudo prospectivo com participação de 26 pacientes com idade entre 51 e 90 anos (média 69,8 anos) com diagnóstico de GPAA ou HO do Departamento de Glaucoma do Centro de Oftalmologia Tadeu Cvintal.

Foram excluídos do estudo os pacientes que apresentaram: uso de lentes de contato, corticosteróides, ciclosporina tópica, lágrimas artificiais, conjuntivite ativa, ceratite, esclerite ou uveíte em qualquer um dos olhos, história de cirurgia intraocular 90 dias antes da introdução da medicação ou durante o curso do estudo, história de doenças inflamatórias oculares, doenças retinianas progressivas como degenerações retinianas ou doença macular relacionada à idade, retinopatia diabética, descolamento de retina, história de hipersensibilidade ao uso de timolol $0,5 \%$ e a inibidores da anidrase carbônica, presença de doenças cardiovasculares, hepáticas ou renais, doenças obstrutivas pulmonares ou asma; que podem dificultar a administração do beta bloqueador, além de gestantes e mulheres em aleitamento materno.

Todos os pacientes tiveram a PIO aferida antes do uso da medicação (dia 0 ) no período da manhã por um único examinador e foram orientados a usar a combinação fixa de timolol 0,5\% e brinzolamida $1 \%$ de 12 / $12 \mathrm{~h}$. Após a introdução da medicação a PIO foi aferida no sétimo dia (dia 7) e no trigésimo dia (dia 30). A PIO foi aferida através de tonometria de aplanação (Goldmann) e os pacientes foram questionados sobre possíveis efeitos colaterais através de entrevista pelo mesmo examinador, com a seguinte questão: Você sentiu alguma alteração ao pingar a medicação prescrita?, diminuindo a possibilidade de sugestão de possíveis efeitos colaterais. 
As variáveis foram resumidas pelas estatísticas descritivas pertinentes: frequência absoluta (n) e relativa (\%) ou média, desvio padrão (dp), mediana, valores mínimo e máximo. Foi aplicado um modelo de Análise de Variância para medidas repetidas para comparar as medidas de PIO ao longo do seguimento. As diferenças entre as avaliações foram localizadas por testes de comparações múltiplas com a correção de Bonferroni.

Foi adotado o nível de significância de $0,05(\mathrm{a}=$ $5 \%$ ) e utilizado o programa estatístico SPSS versão 15.0 for Windows para todas as análises estatísticas.

\section{Resultados}

Foram avaliados cinquenta (50) olhos de vinte e seis (26) pacientes sendo que em dois pacientes hipertensos oculares, a medicação foi instituída em apenas um dos olhos. Foi observada variação estatisticamente significante entre as avaliações da PIO $(\mathrm{p}<0,001)$. Nos testes de comparações múltiplas, a média de PIO no dia 0 mostrou-se significantemente maior do que as médias das demais avaliações ( $\mathrm{p}<0,001$ comparado ao dia 7 e $p<0,001$ comparado ao dia 30) e não foi observada diferença estatisticamente significante entre as médias dos dias 7 e $30(p=1,000)$. A média das pressões intraoculares iniciais foram de $23,8 \mathrm{mmHg}(18$ $30 \mathrm{mmHg}$ ) e após o início da medicação, nos dias 7 e 30 foram de 14,6 e $14,4 \mathrm{mmHg}$ respectivamente, mostrando uma redução média de cerca de $38,65 \%$ no dia 7 e $39,49 \%$ no dia 30 . Os dados são apresentados na tabela 1 e gráfico 1.

Quanto aos efeitos adversos relatados pelos pacientes ao uso da combinação fixa de timolol $0,5 \%$ e brinzolamida $1 \%$, dos 26 pacientes estudados, apenas 4 $(15,4 \%)$ relataram alguma experiência desconfortável. Desses quatro pacientes, dois pacientes $(7,7 \%)$ relataram alguma ardência ao instilar o colírio, um paciente $(3,8 \%)$ relatou algum embaçamento e um paciente $(3,8 \%)$ relatou uma leve queimação.

De todos os pacientes que iniciaram o tratamento com a medicação prescrita, apenas em três (11,5\%) foi necessário acrescentar alguma medicação antiglaucomatosa (Tabela 2), pois não alcançaram a PIO alvo estabelecida de acordo com sua escavação do nervo óptico 30 dias após introduzida a medicação.

\section{DiscuSSÃo}

Cada vez mais as combinações fixas de medicações antiglaucomatosas têm ganhado espaço na prática

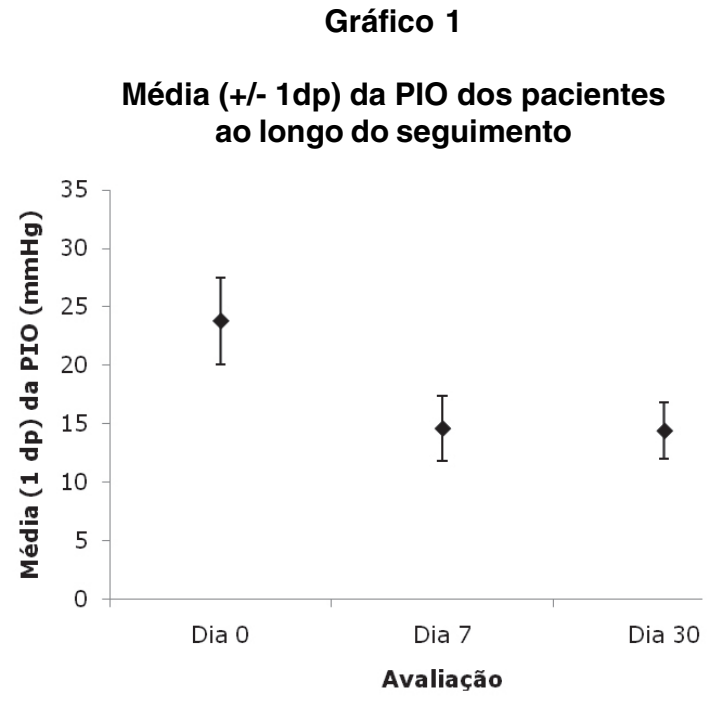

Tabela 1

PIO dos pacientes ao longo do seguimento

\begin{tabular}{lccc}
\hline PIO $(\mathrm{mmHg})(\mathrm{n}=50)$ & \multicolumn{4}{c}{ Avaliação } \\
\cline { 2 - 4 } & Dia 0 & Dia 7 & Dia 30 \\
Média $(\mathrm{dp})$ & $23,8(3,7)$ & $14,6 \quad(2,8)$ & $14,4 \quad(2,4)$ \\
Mediana & 24 & 14 & 15 \\
Mínimo / máximo & $17-30$ & $8-20$ & $8-18$ \\
Pré x Pós & & $\mathrm{p}<0,001 *$ \\
\hline
\end{tabular}

Tabela 2

Efeitos colaterais na amostra de pacientes $(n=26)$

\begin{tabular}{lc}
\hline Efeitos colaterais & n (\%) \\
\hline Queixas & \\
Não & $22(84,6)$ \\
Sim & $4(15,4)$ \\
Queixas (especificação) & \\
Não & $22(84,6)$ \\
Ardência & $2(7,7)$ \\
Embaçamento & $1(3,8)$ \\
Leve queimação & $1(3,8)$ \\
Acrescentou medicação & \\
Não & $23(88,5)$ \\
Sim & $3(11,5)$ \\
\hline
\end{tabular}

clínica diária, por fornecerem maior potência na diminuição da PIO e também porque melhoram a aderência dos pacientes ao tratamento ${ }^{(8,9)}$. Com o surgimento de uma nova combinação no mercado, procuramos avaliar a eficácia e a tolerância dessa nova formulação com pacientes que não usavam nenhuma droga no tratamento do glaucoma. Em nosso estudo obtivemos uma média de di- 
minuição da PIO com a combinação de timolol/ brinzolamida de cerca de $38 \%$, número um pouco maior que em outro estudo que obteve aproximadamente $30 \mathrm{a}$ $33 \%$ de redução da $\mathrm{PIO}^{(1)}$. Nossos pacientes mantiveram a PIO no mesmo patamar nas avaliações de 7 dias e 30 dias, demonstrando eficácia em manter a PIO mais baixa. Quanto ao desconforto dos pacientes ao utilizar a medicação proposta, em nosso estudo uma pequena parcela relatou queixas, apenas quatro pacientes que represen$\operatorname{tam} 15,4 \%$ do total. Desses pacientes, dois queixaram de ardência (7,7\%), um de embaçamento $(3,8 \%)$ e um de leve queimação $(3,8 \%)$. Na literatura encontramos estudos que comparam uma medicação com outra, onde em todas as comparações apresentaram resultados favoráveis à combinação de timolol com brinzolamida ${ }^{(5,10,11)}$.

A combinação de timolol 0,5\% com brinzolamida $1 \%$ mostrou-se eficaz no controle da PIO com índices de redução semelhantes à literatura e mostrou um baixo índice de desconforto nos pacientes acompanhados. No entanto, em nosso estudo, tivemos um número pequeno de pacientes e são necessários mais estudos para evidenciar esses mesmos resultados em longo prazo.

\section{REFERÊNCIAS}

1. Holló G, Bozkurt B, Irkec M. Brinzolamide/timolol fixed combination: a new ocular suspension for the treatment of openangle glaucoma and ocular hypertension. Expert Opin Pharmacother. 2009;10(12):2015-24.

2. Woodward DF, Chen J. Fixed-combination and emerging glaucoma therapies. Expert Opin Emerg Drugs. 2007;12(2):313-27.

3. Ishikawa S, Nakamura Y, Nakamura Y, Sakai H, Sawaguchi S, Terashima K, et al. Efficacy and safety of combination therapy with latanoprost after a change in therapeutic regimen from timolol to brinzolamide in Japanese adult patients with primary open-angle glaucoma and ocular hypertension: open, non-randomized 12-week study. Clinical Ophthalmol. 2008;2(4):703-8.
4. Calugaru D, Calugaru M. [Fixed combinations of glaucoma medications]. Oftalmologia. 2008;52(3):23-31. Review. Romanian.

5. Mundorf TK, Rauchman SH, Williams RD, Notivol R; Brinzolamide/Timolol Preference Study Group. A patient preference comparision of Azarga (brinzolamide/timolol fixed combination) vs Cosopt (dorzolamide/timolol fixed combination) in patients with open-angle glaucoma or ocular hypertension. Clin Ophthalmol. 2008;2(3):623-8.

6. Cvetkovic RS, Perry CM. Brinzolamide: a review of its use in the management of primary open-angle glaucoma and ocular hypertension. Drugs Aging. 2003;20(12):919-47.

7. Croxtall JD, Scott LJ. Brinzolamide/timolol: in open-angle glaucoma and ocular hypertension. Drugs Aging. 2009;26(5):437-46.

8. Spaeth GL, Bernstein P, Caprioli J, Schiffman RM. Control of intraocular pressure and fluctuation with fixed-combination brimonidine-timolol versus brimonidine or timolol monotherapy. Am J Ophthalmol. 2011;151(1):93-9.e4

9. Higginbotham EJ. Considerations in glaucoma therapy: fixed combinations versus their component medications. Clin Ophthalmol. 2010;4:1-9.

10. Manni G, Denis P, Chew P, Sharpe ED, Orengo-Nania S, Coote MA, et al. The safety and efficacy of brinzolamide $1 \% /$ timolol $0.5 \%$ fixed combination versus dorzolamide $2 \% /$ timolol $0.5 \%$ in patients with open-angle glaucoma or ocular hypertension. J Glaucoma. 2009;18(4):293-300.

11. Vold SD, Evans RM, Stewart RH, Walters T, Mallick S. A oneweek comfort study of BID-dosed brinzolamide 1\%/timolol $0.5 \%$ ophthalmic suspension fixed combination compared to BID-dosed dorzolamide $2 \%$ timolol $0.5 \%$ ophthalmic solution in patients with open-angle glaucoma or ocular hypertension. J Ocul Pharmacol Ther. 2008;24(6):601-5.

\section{Endereço para correspondência}

Juliana Almodin

Rua Xavier Curado, no 351 - apto. 131

CEP 04210-100 - Ipiranga (SP), Brasil

Email: Juliana_almodin@hotmail.com 